\title{
Saúde da mulher: enfoque da evidência científica para a prevenção da morbidade e mortalidade materna
}

\section{Woman's health: focusing scientific evidence for prevention of maternal morbidity and mortality}

$\mathrm{Na}$ atualidade a saúde da mulher continua como um tema de interesse, oportuno, pertinente e de debate. Além dos aspectos biológicos e epidemiológicos que sempre nortearam os assuntos associados à saúde dos diferentes grupos populacionais, o atualíssimo enfoque de gênero, introduzido na discussão da saúde das mulheres sob uma perspectiva multidisciplinar e multiprofissional, acrescenta matizes sociais, culturais, éticos, humanísticos e até mesmo antropológicos aos novos velhos temas correlatos, incluindo a gravidez indesejada, o aborto, a violência, a sexualidade, a anticoncepção, a gestação saudável, a forma de nascimento, a qualidade da atenção à saúde, a morbidade associada à gestação, até finalmente a morte materna. Em que pese a importância individual de cada um desses assuntos, parece incrível que nesse início de século seja ainda necessário discutir e, mais que discutir, ainda tentar encontrar caminhos e soluções para enfrentar esse anacrônico e insistente problema da morbidade associada ao mais fisiológico processo da reprodução, o das mulheres engravidarem e parirem com segurança, especialmente nos países em desenvolvimento.

Não é apenas na esfera da saúde pública e dos programas populacionais que se podem recomendar intervenções com o objetivo de reduzir complicações associadas com a gravidez, parto e puerpério para evitar o óbito materno. Além de um componente genérico de saúde geral e condições sociais das mulheres, acredita-se que o adequado atendimento profissional à mulher durante a gestação e, principalmente, durante o parto, desempenhe um papel fundamental na determinação de bons resultados de saúde neste momento. De fato, apenas as melhorias da atenção profissional e institucional ao parto, independentemente das condições das mulheres, é capaz de reduzir significativamente a ocorrência de morbidade materna grave e de mortes maternas. Portanto, dessa responsabilidade não podem eximir-se os profissionais da saúde.

Assim, por exemplo, em relação às boas práticas na atenção obstétrica, as evidências sobre intervenções benéficas no parto, tendo como marco conceitual a chamada "medicina baseada em evidências", deveriam estar disponíveis a partir de estudos de metanálise e revisão sistemática derivados de ensaios controlados aleatorizados que contemplassem especificamente o efeito de cada possível intervenção no parto sobre a saúde das mulheres, em termos de morbidade e mortalidade. ${ }^{1}$ Dado que o interesse específico nesse caso é a morte materna, dois pontos fundamentais devem ser considerados: primeiro, a morbidade materna está intimamente associada à mortalidade materna, pois apresentam determinantes primários comuns; segundo, a morte materna é, em termos absolutos, um evento raro e dificilmente corresponde a uma medida de efeito de grande impacto nos estudos controlados. Tudo isso torna difícil a real avaliação do impacto de intervenções no parto para a redução da mortalidade materna. Entretanto, considerando que a morbidade antecede a mortalidade, é possível se valer da evidência indireta de que qualquer intervenção que consiga reduzir significativamente a morbidade materna deva teoricamente também ser capaz de reduzir a mortalidade materna.

O conceito de evidência científica disponível para justificar a recomendação e/ou o uso de intervenções específicas para se atingir um efeito também específico é cada vez mais difundido no mundo. As revisões sistemáticas e os estudos de metanálise têm a capacidade de sintetizar a experiência compilada de diversos estudos sobre uma determinada intervenção, que permite um efeito resumo comum. A evidência científica pode ser considerada tanto mais forte, quanto mais poderoso tiver sido o desenho de estudo de onde o resultado saiu para gerar a evidência. Assim, por ordem decrescente, têm maior capacidade de gerar fortes evidências os estudos de revisões sistemáticas, ensaios controlados aleatorizados, estudos de coorte, estudos caso-controle, séries de casos e, apenas por último, a opinião de expertos, baseada em sua experiência pessoal. Na dependência do nível da evidência disponível, classificam-se três tipos de recomendação para utilização da intervenção: A, revisões sistemáticas, ensaios controlados aleatorizados e estudos de coorte; B, estudos caso-controle e C, série de casos e opinião de expertos, também em ordem decrescente, que atualmente passam a orientar a maior parte dos guias e manuais de condutas sobre práticas médicas. 2,3 
Especificamente abordando as intervenções benéficas para o parto, no sentido de se obter o efeito de redução da morbidade e mortalidade materna, algumas publicações recentes têm avaliado criticamente a questão, disponibilizando informações úteis, tanto sob o ponto de vista individual, para o cuidado de cada mulher, como principalmente no nível coletivo, quando se decide por intervenções e procedimentos recomendados em manuais ou protocolos de orientação clínica de instituições, sociedades médicas ou da saúde pública de cidades ou países. $1,3-5$

Assim, existem alguns aspectos relativos ao conhecimento baseado em evidências científicas sobre uma série de intervenções relacionadas ao parto e que, potencialmente, podem resultar em complicações capazes de caracterizar uma morbidade que, se não adequadamente conduzida e tratada, pode evoluir para o óbito da mulher. Entre as principais intervenções, sobressaem a atenção institucional ao parto, o atendimento profissional capacitado, utilização de parteiras tradicionais em determinados contextos, uso de tecnologias apropriadas incluindo o partograma, local do parto, posição para o parto, uso de episiotomia, tipo de parto, uso de ocitócicos na fase ativa do parto, realização de esforços de puxo no período expulsivo, manejo da dequitação e profilaxia da hemorragia puerperal. Tais intervenções, seguramente, poderiam ser mais e melhor abordadas nos manuais de conduta obstétrica de nossos países.

Especificamente abordando as tecnologias obstétricas apropriadas para lidar com complicações maternas, muitas são as evidências sobre o conjunto de procedimentos, condutas e terapêuticas mais eficientes para tais situações, especialmente aquelas relativas às mais comuns ocorrências dos países ou regiões com menos recursos para a saúde. Vários exemplos poderiam ser destacados nesta categoria. Provavelmente uma das situações que têm como conseqüência maior morbidade em áreas com parcos recursos é a do parto obstruído, responsável ainda por uma parcela significativa da mortalidade materna no mundo. Entretanto, existem muitas evidências sobre intervenções de fato efetivas para sua prevenção ou manejo, como é o caso do uso rotineiro recomendado do partograma e da disponibilização do acesso ao parto cirúrgico, pressupondo equipamentos e profissionais adequados ao seu manejo. Outras situações que podem ser lembradas são a prevenção da convulsão na eclâmpsia pela utilização do sulfato de magnésio, a prevenção da sepse puerperal pelo uso de antibioticoterapia profilática sistemática nos casos de cesárea, o manejo adequado da malária associada à gestação, e também a prevenção da hemorragia puerperal pela utilização dos procedimentos do manejo ativo do terceiro período, incluindo o uso de ocitocina ou misoprostol como drogas uterotônicas, a tração controlada do cordão umbilical e a massagem uterina abdominal suave até a dequitação.6,7

Outro ponto que pode ser enfocado é o das auditorias sobre procedimentos e resultados em atenção à saúde perinatal. Essencialmente abordando os resultados de diminuição da mortalidade materna, parecem ter pequena efetividade os inquéritos confidenciais e retrospectivos sobre a ocorrência de mortes maternas, de morbidade materna grave e de determinados procedimentos como cesárea ou episiotomia. Por outro lado, demonstram estar associadas a maior efetividade das intervenções com a finalidade da redução das mortes maternas, políticas públicas especificamente dirigidas à redução das mortes maternas, projetos para reduzir na população as barreiras financeiras para uma assistência materna de qualidade, a disponibilidade de serviços maternos acessíveis e sem custo, o aumento do número de obstetrizes qualificadas para a adequada atenção ao parto, além do desenvolvimento de sistemas de vigilância epidemiológica no futuro para a prevenção do óbito materno. ${ }^{8}$

Nesse sentido, um novo enfoque de estudar e talvez de monitorizar a ocorrência de mortes maternas pode ser representado pelo desenvolvimento do conceito de near miss materna, ou seja, de morbidade materna grave que representaria o estágio imediatamente anterior à ocorrência do óbito. Registro vital incompleto, sub-notificação e dispersão territorial são fatores que dificultam o estudo acurado da mortalidade materna e favorecem seu negligenciamento, estimulando a busca de um indicador que possa contribuir para o combate à morte materna. Considerando que, nos países desenvolvidos, a morte materna tornou-se um evento cada vez mais raro, aumenta o interesse em torno da morbidade obstétrica grave, de near-miss, em que mulheres apresentam complicações potencialmente letais durante a gravidez, parto ou puerpério e somente sobrevivem devido a um bom cuidado hospitalar ou à sorte. ${ }^{9} \mathrm{O}$ estudo de tais casos é de grande valor para compreender os determinantes da morbimortalidade materna, por sua maior freqüência e capacidade de fornecer informações que os casos de óbito materno e também porque a própria mulher pode ser fonte de dados.

A auditoria sobre a ocorrência desta morbidade materna grave, ou seja, dos casos de near miss, sobretudo se realizada de maneira rotineira, prospectiva e com o objetivo de funcionar como um verdadeiro sistema de vigilância epidemiológica, provavelmente poderia estar associada à obtenção de um melhor resultado, se medidas adequadas tomadas em tempo adequado fossem instituídas com o propósito de alterar a seqüência da cadeia de eventos mórbidos que culminariam com o óbito.10-12 Nessa direção, à medida que a sobrevivência nos casos 
de near-miss decorre em grande parte do cuidado oferecido, a avaliação desses casos também tem sido utilizada para determinar a qualidade da atenção obstétrica, auxiliando na compreensão dos casos de morte materna. A ausência de um planejamento detalhado na oferta de cuidados pode levar à ocorrência de retardos na implementação das medidas necessárias, estando esses retardos associados ao desfecho materno-fetal desfavorável.13

Ainda que fatores políticos, econômicos, sociais e culturais possam ser também determinantes da qualidade em saúde, especialmente da saúde das mulheres, não existem mais dúvidas de que os profissionais da área muito podemos fazer tecnicamente para melhorar a qualidade - de saúde e de vida - dessas mulheres, mesmo que não exerçamos nenhum tipo de pressão ou influência para mudanças de políticas programáticas junto a nossos governos e governantes.

\section{José Guilherme Cecatti}

Departamento de Tocoginecologia. Universidade Estadual de Campinas Rua Alexander Fleming, 101, Campinas, SP, Brasil. CEP: 13.083-881 Fone: 019-37889482 e-mail: cecatti@unicamp.br

\section{Referências}

1. WHO (World Health Organization). The WHO Reproductive Health Library 2004 (7). [CD ROM]. Geneva; 2004.

2. Mulrow $\mathrm{CD}$. The medical review article: state of the science. Ann Intern Med 1997; 106: 185-8.

3. Ministério da Saúde. Parto, aborto e puerpério. Assistência humanizada à mulher. Brasília (DF); 2001

4. De Brouwere V, Van Lerberghe W, editors. Safe motherhood strategies: a review of the evidence: Stud Health Serv Organ Pol 2001 (17)

5. Gay J, Hardee K, Judice N, Agarwal K, Fleming K, Hairston A, Walker B, Wood M. What works: a policy and program guide to the evidence on family planning, safe motherhood and STI/HIV/AIDS interventions. Module 1: Safe Motherhood. Policy Project March 2003.

6. Bergstrom S. Appropriate obstetric technologies to deal with maternal complications. In: De Brouwere V, Van Lerberghe $\mathrm{W}$, editors. Safe motherhood strategies: a review of the evidence. Stud Health Serv Organ Pol 2001; 17: 175-94.

7. Tsu VD. New and underused technologies to reduce maternal mortality. Lancet 2004; 363: 75-6.
8. Ronsmans C. What is the evidence for the role of audits to improve the quality of obstetric care. In: De Brouwere V, Van Lerberghe W, editors. Safe motherhood strategies: a review of the evidence. Stud Health Serv Organ Pol 2001; 17: 207-28.

9. Stones W, Lim W, Al-Azzawi F, Kelly M. An investigation of maternal morbidity with identification of life-threatinig 'near miss' episodes. Health Trends 1991; 23: 13-5.

10. Mantel GD, Buchmann E, Rees H, Pattinson RC. Severe acute maternal morbidity: a pilot study of a definition for near miss. Br J Obstet Gynaecol 1998; 105: 985-90.

11. Pattinson RC, Hall M. Near misses: a useful adjunct to maternal death enquiries. Br Med Bull 2003; 67: 231-43.

Geller SE, Rosenberg D, Cox SM, Kilpatrick S. Defining a conceptual framework for near-miss maternal morbidity. J Am Med Womens Assoc 2002; 57: 135-9.

12. Maine D, Akalin MZ, Ward VM, Kamara A. The design and evaluation of maternal mortality programs. New York: Centre for Population and Family Health, School of Public Health, Columbia University; 1997. 